\title{
BRIEF REPORT Diffuse Intrinsic Pontine Glioma Biopsy: A Single Institution Experience
}

\author{
Zhihong J. Wang, MD, PhD, ${ }^{1}$ Latha Rao, MD, ${ }^{1}$ Kanta Bhambhani, MD, ${ }^{1}$ Kathy Miller, $\mathrm{BS}^{1}{ }^{1}$ Janet Poulik, MD, ${ }^{2}$ \\ Deniz Altinok, $\mathrm{MD}^{3}$ and Sandeep Sood, $\mathrm{MD}^{4}$
}

Tumor biopsy is rarely performed in diffuse intrinsic pontine glioma (DIPG) due to the presumed risk of surgical complications, although data on the surgery related morbidity of DIPG biopsy is sparse. We performed a retrospective review on 22 consecutive cases of DIPG diagnosed from 2002 to 2012 at Children's Hospital of Michigan, 15 of which underwent biopsy. Transient new or worsening neurological deficits were observed in three of 15 cases following surgery. No surgery related mortality or permanent deficit was observed, and the mean overall survival was $10.4 \pm 3.8$ months. Undergoing biopsy did not adversely affect the outcome. Pediatr Blood Cancer 2015;62:163-165. (c) 2014 Wiley Periodicals, Inc.

Key words: astrocytoma; biopsy; DIPG

\section{INTRODUCTION}

Diffuse Intrinsic Pontine Glioma (DIPG) remains a leading cause of death for children with brain tumors. DIPG is unresectable because of its location and infiltrative nature. The role of biopsy for patients with DIPG has been controversial [1-4]. In 1993, Albright reported the results of the Children's Cancer Group (CCG) 9882 DIPG study and concluded that magnetic resonance imaging (MRI) was adequate in making the diagnosis, and tissue diagnosis did not alter the treatment nor did it impact outcome [1]. Based on this conclusion and the presumed risk of surgical complications, tumor biopsy has been rarely performed.

There has been no improvement in the outcome for patients with DIPG in the past 30 years despite decades of clinical trials. The lack of primary tumor materials from not performing biopsy had made preclinical research difficult and hindered the development of new therapies. An effort has been made to acquire post mortem samples [5-8]. While the information generated from autopsy materials has been very helpful in confirming the clinical diagnosis and understanding tumor biology, it is often confounded by the effect of prior radiation treatment and limited by the difficulty in collecting tissues in a timely fashion. In addition, DIPG at diagnosis may be molecularly distinct from that identified at the time of autopsy [8]. While the necessity for tumor tissue at diagnosis has been increasingly recognized, the morbidity associated with the biopsy is not well defined due to the rarity of this procedure performed, so the debate on whether to perform a biopsy upfront continues $[1,2,4]$. The aim of this study was to retrospectively review our experience of surgical biopsy of DIPG.

\section{METHODS}

Twenty-two consecutive cases of DIPG diagnosed and treated from 2001 to 2012 at the Children's Hospital of Michigan (CHM) were identified from the institutional database and reviewed retrospectively. Because of the small number of cases, all statistics are descriptive. This project was approved by the Human Investigation Committee of Wayne State University.

\section{RESULTS}

Among the 22 cases with a diagnosis of DIPG, 12 were males and 10 were females. The age at presentation ranged from
20 months to 16 years. Risks of procedure were discussed with patient's guardian prior to the procedure, and a biopsy was performed on 15 of those 22 cases. The characteristics and outcomes of these 15 cases are summarized in Table I.

All patients underwent a stealth guided stereotactic biopsy of the lesion using a posterior fossa transcerebellar peduncle approach. Biopsies were done using Medtronic Biopsy Needle $\mathrm{Kit}^{\mathrm{B}}$ (Medtronic Navigation Inc, 826 Coal Creek Circle, Louisville, CO 80027) under stereotactic guidance. Dexamethasone was started prior to or right after surgery on all patients. No intraoperative complications or surgery-related mortality was observed, and no permanent deficits were noted. Transient new or worsening neurological deficits were recorded in three of 15 patients. Case 11 presented with a history of ataxia and cranial nerve palsy with MRI findings typical of DIPG (Fig. 1A). Her symptoms worsened following a biopsy, but started to improve two weeks after starting radiation therapy and completely resolved at the completion of treatment, with significant reduction in size of the tumor in the follow up MRI (Fig. 1B). A transient increase in weakness of the lower extremities and worsening of speech was observed in Case 13, and a transient left facial palsy was recorded in Case 14.

All biopsies were successful, in that adequate tumor tissue was collected for histopathological diagnosis and immunohistochemical (IHC) staining. All patients had astrocytoma. Thirteen had highgrade astrocytoma and two patients had a low-grade astrocytoma. IHC staining for $\mathrm{p} 53$ and epidermal growth factor receptor (EGFR) was performed in eight cases and was positive in six cases for each marker (Table I).

As for the treatment and outcome, Case 5 received supportive care only, and Case 14 was transferred to another center following

\footnotetext{
${ }^{1}$ Pediatric Hematology Oncology, The Carman and Ann Adams Department of Pediatrics, Wayne State University, 3901 Beaubien Street, Detroit, Michigan 48201; ${ }^{2}$ Pathology, Wayne State University, Detroit, Michigan; ${ }^{3}$ Radiology, Wayne State University, Detroit, Michigan; ${ }^{4}$ Neurosurgery, Children's Hospital of Michigan, Wayne State University, Detroit, Michigan

Conflict of interest: Nothing to report.

* Correspondence to: Zhihong Wang, Division of Pediatric Hematology Oncology, Children's Hospital of Michigan, 3901 Beaubien Street, Detroit, MI 48201. E-mail: jwang2@med.wayne.edu
}

Received 21 March 2014; Accepted 20 July 2014 
TABLE I. DIPG Case Summary

\begin{tabular}{|c|c|c|c|c|c|c|c|c|}
\hline & $\begin{array}{c}\text { Age } \\
\text { (Years) }\end{array}$ & Sex & Presenting symptoms & $\begin{array}{l}\text { New } \\
\text { deficits }\end{array}$ & $\begin{array}{l}\text { Astrocytoma } \\
\text { WHO grade }\end{array}$ & $\begin{array}{l}\text { Survival } \\
\text { (Months) }\end{array}$ & p53 & EGFR \\
\hline 1 & 4 & $\mathrm{~F}$ & $\begin{array}{l}\text { ataxia, left facial \& } 6 \text { th nerve palsy, } \\
\text { right extremity weakness }\end{array}$ & No & I & 10 & N/A* & N/A \\
\hline 2 & 3 & $\mathrm{~F}$ & ataxia, right facial palsy, slurred speech & No & II & 9 & N/A & N/A \\
\hline 3 & 13 & M & headache, diplopia & No & III & 7 & N/A & N/A \\
\hline 4 & 12 & M & ataxia, slurred speech, right extremity weakness & No & III & 8 & N/A & N/A \\
\hline 5 & 4 & M & headache, ataxia, vomiting & No & III & 4 & positive & positive \\
\hline 6 & 7 & M & ataxia, 6th nerve palsy & No & IV & 18 & N/A & N/A \\
\hline 7 & 9 & M & ataxia & No & III & 13 & positive & positive \\
\hline 8 & 16 & M & incidental finding followed by headache & No & IV & 9 & negative & positive \\
\hline 9 & 14 & M & ataxia, slurred speech & No & III & 10 & positive & negative \\
\hline 10 & 9 & $\mathrm{~F}$ & ataxia, headache, vomiting & No & III & 16 & negative & positive \\
\hline 11 & 4 & $\mathrm{~F}$ & ataxia, left facial palsy, slurred speech & Yes & IV & 12 & positive & positive \\
\hline 12 & 7 & M & ataxia, right facial palsy, left extremity weakness & No & IV & 9 & positive & negative \\
\hline 13 & 7 & $\mathrm{~F}$ & ataxia, ptosis, slurred speech & Yes & III & 7 & N/A & N/A \\
\hline 14 & 15 & M & headache & Yes & IV & N/A & positive & positive \\
\hline 15 & 8 & $\mathrm{~F}$ & ataxia, left facial palsy, bilateral weakness & No & III & 14 & N/A & N/A \\
\hline
\end{tabular}

${ }^{*}$ N/A: not available.

diagnosis. All other patients received involved-field external beam radiation and several patients received chemotherapy. All patients died of disease progression with a mean overall survival of $10.4 \pm 3.8$ months. The Case 1 was a 4 -year-old female who presented with a history of ataxia, cranial nerve palsy, and lower extremity weakness for 5 days with a MRI findings typical of DIPG (Fig. 1C). Tumor histology was consistent with grade I astrocytoma. She responded to radiation therapy initially with improvement of
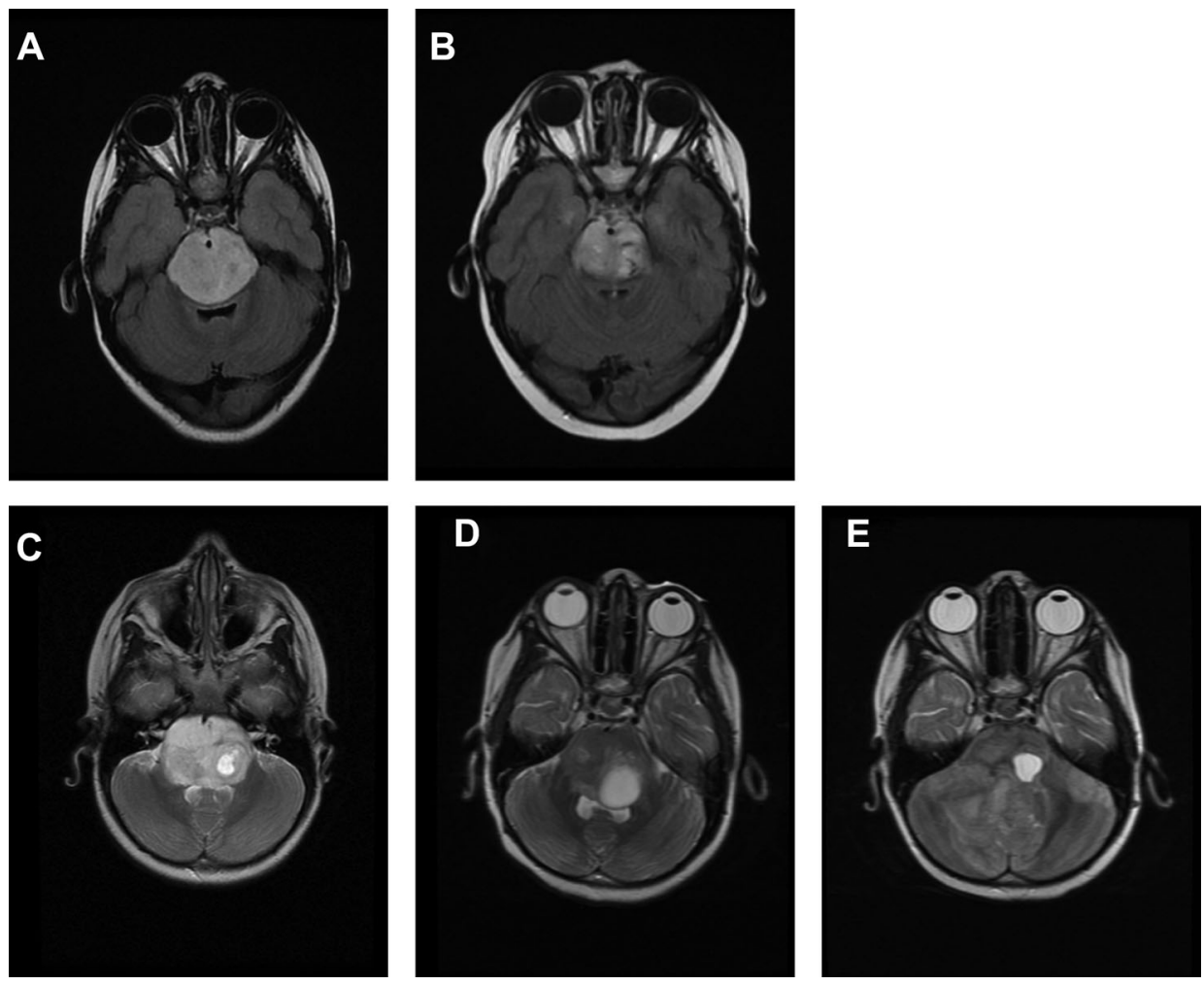

Fig. 1. MRI images at diagnosis and at follow up for Case 11 (A and $\mathrm{B}$ ) and Case 1 (C, D and E)). Axial T2 Flair image showing diffuse, expansile, and infiltrating hyperintense pontine mass at diagnosis (A), which was significantly decreased in size following completion of radiation therapy (B). Axial T2W images at diagnosis (C) showed intraaxial, infiltrating, and expansile mass lesion involving center of the pons, which was decreased in size and intensity following radiation therapy (D), but significantly increased in size associated with transtentorial ascenden herniation 5 months later $(\mathbf{E})$. 
her symptoms and a decrease in size of the tumor by MRI (Fig. 1D) but progressed 5 months later (Fig. 1E) then died 10 months following diagnosis.

\section{DISCUSSION}

The diagnosis of DIPG is generally based on clinical and radiological findings as biopsy has been rarely performed. In an earlier experience from our institution, a patient suspected of DIPG by the radiological findings was diagnosed with a primitive neuroectodermal tumor (PNET) based on the histology after biopsy. Since the management and prognosis for PNET versus DIPG is very different, tumor biopsy had been performed in our institution on the majority of patients with radiological findings suggestive of DIPG. In our cohort, there was no intraoperative complication or surgeryrelated mortality. The transient new or worsening neurological deficits observed in three patients could be due to cerebral edema following procedures instead of surgical injuries, since all resolved within a short period of time. In a recent French study on pediatric DIPG, transient worsening of neurological deficit was observed in only four out of 90 patients, suggesting stereotactic biopsy for children with DIPG was a safe procedure [2], and similar results were reported by others [8-11]. The overall survival in our cohort was comparable to that reported in the literature [12], suggesting that undergoing a biopsy did not adversely impact the outcome. The diagnostic yield was $100 \%$ in our series, and the majority of patients had a high-grade astrocytoma, consistent with that in the published reports $[8,9,13]$. Although the histologic diagnosis in Case 1 was consistent with a low-grade glioma, her clinical course then imaging findings were indistinguishable from those of high-grade glioma, therefore, sampling error or tumor heterogeneity could not be ruled out without examining the entire tumor.

Since tumor biopsy in DIPG is rarely performed, we hope to share our experience with the pediatric oncology community. Whether to perform the biopsy upfront in DIPG has been an ongoing debate, and given the small number of cases in our series, we don't feel any conclusion can be drawn. While it is generally felt that biopsy is not clinically indicated for DIPG with classic MRI findings, postmortem evaluation has revealed a histological diagnosis of PNET in as many as $22 \%$ of patients thought to have
DIPG $[6,14]$ in which long-term survival has been reported with a combined modality therapy $[14,15]$. The relative low-risk associated with the DIPG biopsy in our experience and in the literature, including the recent prospective experience in specialized centers [8], suggests that biopsy is generally safe if it is performed by skilled neurosurgeons. On the other hand, there has been no definite treatment identified to be effective to certain group of patients. Current census is that until we have proven that the results of the biopsy will change the treatment and provide the direct benefit to patients with DIPG, this procedure should be offered only through clinical trials, or to those cases atypical in clinical presentations or imaging findings.

\section{REFERENCES}

1. Hankinson TC, Campagna EJ, Foreman NK, et al. Interpretation of magnetic resonance images in diffuse intrinsic pontine glioma: A survey of pediatric neurosurgeons. J Neurosurg Pediatr 2011;8: $97-102$

2. Puget $\mathrm{S}$, Blauwblomme T, Grill J. Is biopsy safe in children with newly diagnosed diffuse intrinsic pontine glioma? Am Soc Clin Oncol Educ Book 2012;32:629-633.

3. Albright AL, Packer RJ, Zimmerman R, et al. Magnetic resonance scans should replace biopsies for the diagnosis of diffuse brain stem gliomas: A report from the Children's Cancer Group. Neurosurgery 1993:33:1026-1029; discussion 1029-1030

4. MacDonald TJ. Diffuse intrinsic pontine glioma (DIPG): Time to biopsy again? Pediatr Blood Cancer 2012;58:487-488.

5. Khuong-Quang DA, Buczkowicz P, Rakopoulos P, et al. K27M mutation in histone H3. 3 defines clinically and biologically distinct subgroups of pediatric diffuse intrinsic pontine gliomas. Acta Neuropathol 2012;124:439-447.

6. Angelini $\mathrm{P}$, Hawkins $\mathrm{C}$, Laperriere $\mathrm{N}$, et al. Post mortem examinations in diffuse intrinsic pontine glioma: Challenges and chances. J Neurooncol 2011;101:75-81.

7. Caretti V, Jansen MH, van Vuurden DG, et al. Implementation of a multi-institutional diffuse intrinsic pontine glioma autopsy protocol and characterization of a primary cell culture. Neuropathol Appl Neurobiol 2013;39:426-436.

8. Grill J, Puget S, Andreiuolo F, et al. Critical oncogenic mutations in newly diagnosed pediatric diffuse intrinsic pontine glioma. Pediatr Blood Cancer 2012;58:489-491.

9. Cage TA, Samagh SP, Mueller S, et al. Feasibility, safety, and indications for surgical biopsy of intrinsic brainstem tumors in children. Childs Nerv Syst 2013;29:1313-1319.

10. Pirotte BJ, Lubansu A, Massager N, et al. Results of positron emission tomography guidance and reassessment of the utility of and indications for stereotactic biopsy in children with infiltrative brainstem tumors. J Neurosurg 2007;107:392-399.

11. Roujeau T, Machado G, Garnett MR, et al. Stereotactic biopsy of diffuse pontine lesions in children. J Neurosurg 2007;107:1-4.

12. Cohen KJ, Heideman RL, Zhou T, et al. Temozolomide in the treatment of children with newly diagnosed diffuse intrinsic pontine gliomas: A report from the Children's Oncology Group. Neuro Oncol 2011; 13:410-416.

13. Sufit A, Donson AM, Birks DK, et al. Diffuse intrinsic pontine tumors: A study of primitive neuroectodermal tumors versus the more common diffuse intrinsic pontine gliomas. J Neurosurg Pediatr 2012;10:81-88.

14. Robison NJ, Kieran MW. Diffuse intrinsic pontine glioma: A reassessment. J Neurooncol 2014:119:7-15

15. Fangusaro J, Finlay J, Sposto R, et al. Intensive chemotherapy followed by consolidative myeloablative chemotherapy with autologous hematopoietic cell rescue (AuHCR) in young children with newly diagnosed supratentorial primitive neuroectodermal tumors (sPNETs): Report of the Head Start I and II experience. Pediatr Blood Cancer 2008;50:312-318. 